\section{IN BRIEF}

\section{SPONDYLOARTHRITIS}

\section{IL-17A blockade effective for AS}

Ixekizumab (an anti-IL-17A monoclonal antibody) is superior to placebo for the treatment of DMARD-naive patients with ankylosing spondylitis (AS), according to the 16-week results of the COAST-V phase III randomized controlled trial (341 patients, randomized 1:1:1:1). More patients achieved the stringent Assessment of Spondyloarthritis International Society $40 \%$ response primary endpoint when treated with ixekizumab (every 2 weeks $=52 \%$; every 4 weeks $=48 \%$ ) than with placebo (every 2 weeks $=18 \%$ ), results that were not inferior to treatment with a TNF inhibitor (every 2 weeks $=36 \%$ ).

ORIGINAL ARTICLE van der Heijde, D. et al. Ixekizumab, an interleukin-17A antagonist in the treatment of ankylosing spondylitis or radiographic axial spondyloarthritis in patients previously untreated with biological disease-modifying anti-rheumatic drugs (COAST-V): 16 week results of a phase 3 randomised, double-blind, active-controlled and placebocontrolled trial. Lancet https://doi.org/10.1016/S0140-6736(18)31946-9 (2018)

\section{$\Rightarrow$ THERAPY}

\section{Infection risk with tofacitinib plus glucocorticoid}

The increased risk of infection with herpes zoster virus in patients with rheumatoid arthritis (RA) treated with the Janus kinase inhibitor tofacitinib is further increased by the concomitant use of glucocorticoids. In a study of 8,030 patients with RA in the USA treated with tofacitinib, additional glucocorticoid use nearly doubled the risk of herpes zoster infection $(\mathrm{HR}=1.96,95 \% \mathrm{Cl} 1.33-2.88)$, whereas additional methotrexate use did not increase the risk of herpes zoster infection ( $\mathrm{HR}=0.99,95 \% \mathrm{Cl} 0.64-1.54)$.

ORIGINAL ARTICLE Curtis, J. R. et al. Herpes zoster in tofacitinib: risk is further increased with glucocorticoids but not methotrexate. Arthritis Care Res. https://doi.org/10.1002/ acr.23769 (2018)

\section{$\Rightarrow$ PAEDIATRIC RHEUMATOLOGY}

\section{Treat-to-target possible for JIA}

Following a treat-to-target strategy resulted in remission at 24 months regardless of which of three different treatment plans (DMARD alone, DMARD plus prednisolone or DMARD plus etanercept) was used in a randomized, single-blinded study of 94 DMARD-naive patients with juvenile idiopathic arthritis (IIA). When averaged across the three groups, at 24 months $71 \%$ of patients had achieved inactive disease and $39 \%$ of patients had achieved drug-free inactive disease. Time-to-flare following DMARD discontinuation did not differ between the groups.

ORIGINAL ARTICLE Hissink Muller, P. et al. Treat to target (drug-free) inactive disease in DMARD-naive juvenile idiopathic arthritis: 24-month clinical outcomes of a three-armed randomised trial. Ann. Rheum. Dis. https://doi.org/10.1136/annrheumdis-2018-213902 (2018)

\section{$\Rightarrow$ OSTEOARTHRITIS}

\section{Fat pad MRI signal linked to OA progression}

Individuals with a large volume of effusion-synovitis and/or an alteration in infrapatellar fat pad signal intensity on MRI have an increased risk of developing accelerated knee osteoarthritis (OA). Using participants from the Osteoarthritis Initiative, three groups of individuals were compared ( $n=125$ per group): those with accelerated knee OA; those with knee OA; and those without knee OA. Patients with accelerated knee OA had an average of $44 \%$ more effusion-synovitis volume than those with knee OA or no knee OA 2 years before disease onset, and $>50 \%$ had a change in infrapatellar fat pad signal intensity on MRI. ORIGINAL ARTICLE Davis, J. E. et al. Effusion-synovitis and infrapatellar fat pad signal intensity alteration differentiate accelerated knee osteoarthritis. Rheumatology https://doi.org/10.1093/rheumatology/key305 (2018)

\title{
Double-negative B cells
}

New research published in Immunity identifies a pathogenic function for the extra-follicular B cell differentiation pathway in patients with systemic lupus erythematosus (SLE).

$\mathrm{B}$ cells that undergo class switching but lack expression of immunoglobulin D and the memory marker CD27 are commonly referred to as doublenegative (DN) B cells. These cells seem to serve different functions in the context of different diseases. In patients with HIV or malaria they have been characterized as having either an exhausted or anergic phenotype, respectively; however, a function in autoimmune disease is less clear.

To understand how these cells might contribute to autoimmunity, the researchers of the new study isolated and characterized DN B cells from two cohorts of patients with SLE. Patients in the SLE-2 cohort $(n=50)$ had higher disease activity, serum autoantibody titres and frequency of lupus nephritis than patients in the SLE-1 cohort $(n=40)$. Although the DN B cell population was expanded relative to healthy individuals in both cohorts, the DN B cell population from the SLE-2 cohort was highly enriched for a subset of DN B cells that lack the follicular homing marker CXCR5. Unlike CXCR5 ${ }^{+}$

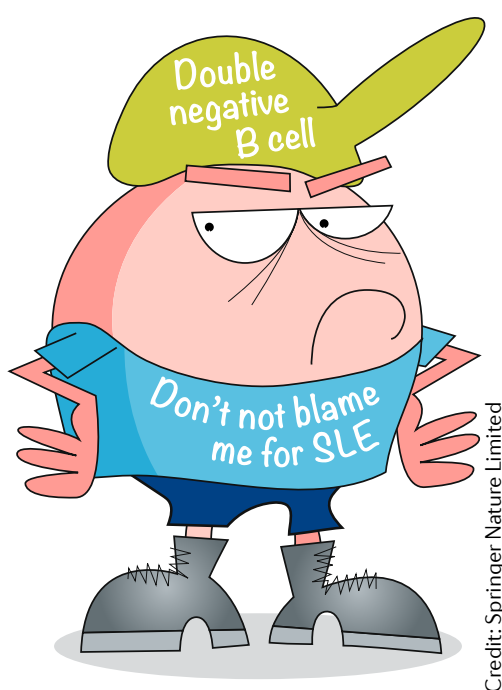

cells (called DN1 cells), these extra-follicular cells (called DN2 cells) were also more numerous in African-American individuals relative to people of other ethnic backgrounds, which might explain the high prevalence or severity of SLE in this population.

Using flow cytometry, epigenomics and transcriptomics, the researchers characterized the DN2 cells, showing that these cells are probably a unique lineage and that in patients with SLE they are frequently specific for known autoantigens.

"The DN2 cells show some differentiation characteristics of cells on the way to becoming a plasmablast, and are thus distinct from naive B cells," says Steve Nutt, a B cell differentiation expert not associated with the new study.

So why do these autoreactive cells proliferate so much in patients with SLE?

"Cause and effect are extremely difficult to separate in a complex systemic autoimmune disease such as SLE," cautions Nutt. "However, the DN2 cells show a striking hyper-plasmablast differentiation response to TLR7 agonists and a role for TLR7 hyper-responsiveness in the promotion of pathology is supported by studies in animal models."

Whether these findings will translate into the development of therapeutics that target DN2 $B$ cells is unclear, and Nutt points out that presently we have no way of depleting the DN2 B cell subset specifically. However, he is optimistic that these new data will be clinically important. "Detecting the frequency of DN2 $B$ cells in patients may be useful for patient stratification and monitoring as well as provide a biomarker for clinical intervention studies," he says.

\section{Nicholas J. Bernard}

ORIGINAL ARTICLE Jenks, S. A. et al. Distinct effector $B$ cells induced by unregulated Toll-like receptor 7 contribute to pathogenic responses in systemic lupus erythematosus. Immunity $\mathbf{4 9}$ 725-739.e6 (2018) 